\title{
Structural and Chemical Characterisation of Metal and Metal Oxide Nanoparticles.
}

\author{
A.I.Kirkland*, S.J.Haigh,*** N.P.Young*, H Sawada*** and K. Takayanagi,**** \\ * Department of Materials, University of Oxford, Parks Road, Oxford, OX1 3PH, UK \\ ** Now at: School of Materials, University of Manchester, Grosvenor Street, Manchester, \\ M1 7HS, UK \\ *** JEOL Ltd.1-2 Mushashino 3-Chome, Akishima, Tokyo 196, Japan. \\ **** Department of Physics, Tokyo Institute of Technology, 2-12-1-H-51 Oh-okayama, \\ Meguro-ku, Tokyo 152-8551, Japan, CREST Japan Science and Technology Corporation, \\ Kawaguchi, Saitama 332-0012 Japan.
}

Morphology and surface structure in nanocrystalline metals and metal oxides is a key feature in determining their fundamental properties and in particular their catalytic activity and selectivity.

This paper will discuss the characterisation metal and metal oxide nanoparticles using aberration corrected electron microscopy and exit wavefunction reconstruction. The correlation of this data with thermodynamic models of morphology and surface structure to produce quantitative phase diagrams that predict stable forms as a function of particle size and temperature for nanoscale systems will also be described.

With reference to the above a number of representative example systems will be highlighted. Nanocrystalline gold has also been demonstrated to show specific catalytic activity at small particle size. In situ imaging experiments (FIG 1) at elevated and depressed temperatures provide direct evidence for morphological changes that can be correlated with theoretical models $[1,2]$ to construct the first quantitative nanoscale phase diagram relating local structure and morphology to temperature and particle size in this system (FIG 2).

Cerium dioxide nanocrystals, have attracted considerable interest due to their properties that lead to applications as efficient oxygen buffers in three-way automotive catalysts. These buffers are essential in stabilizing the air-to-fuel ratio necessary to achieve simultaneous conversion of $\mathrm{NO}, \mathrm{CO}$, and hydrocarbons during both the fuel-lean and fuel-rich stages of the combustion cycles $[3,4]$. Aggregation behaviour in this system is driven by the compatibility of the shape of individual nanoparticles (characterized by the relative fractions of different crystallographic facets and their surface terminations) and can be substantially reduced by control of the shape of individual nanoparticles during synthesis. Experimental and theoretical data has been used to characterise the surface structure and chemistry within this system [5], which has lead to a detailed understanding of the factors controlling morphology and the frequently observed oxygenated surface termination which is driven by migration of oxygen defects from the bulk of the particle.

\section{References}

[1] M.A. van Huis, N.P. Young, G. Pandraud, J.F. Creemer, D. Vanmaekelbergh, A.I. Kirkland, H.W. Zandbergen, Adv. Mater., 21, (2009) 4992

[2] N. P. Young, M.A. van Huis, H.W. Zandbergen, H. Xu, A.I. Kirkland, Ultramicroscopy, 110, (2010) 506.

[3] L. A. J.Garvie, P. R. Buseck, J. Phys. Chem. Solids 60, (1999) 1943

[4] D. J. M. Bevan, J. Kordis, J. Inorg. Nucl. Chem. 26, (1964) 1509.

[5] A. S. Barnard, A. I. Kirkland. Chem. Mater. 20, (2008) 5460 

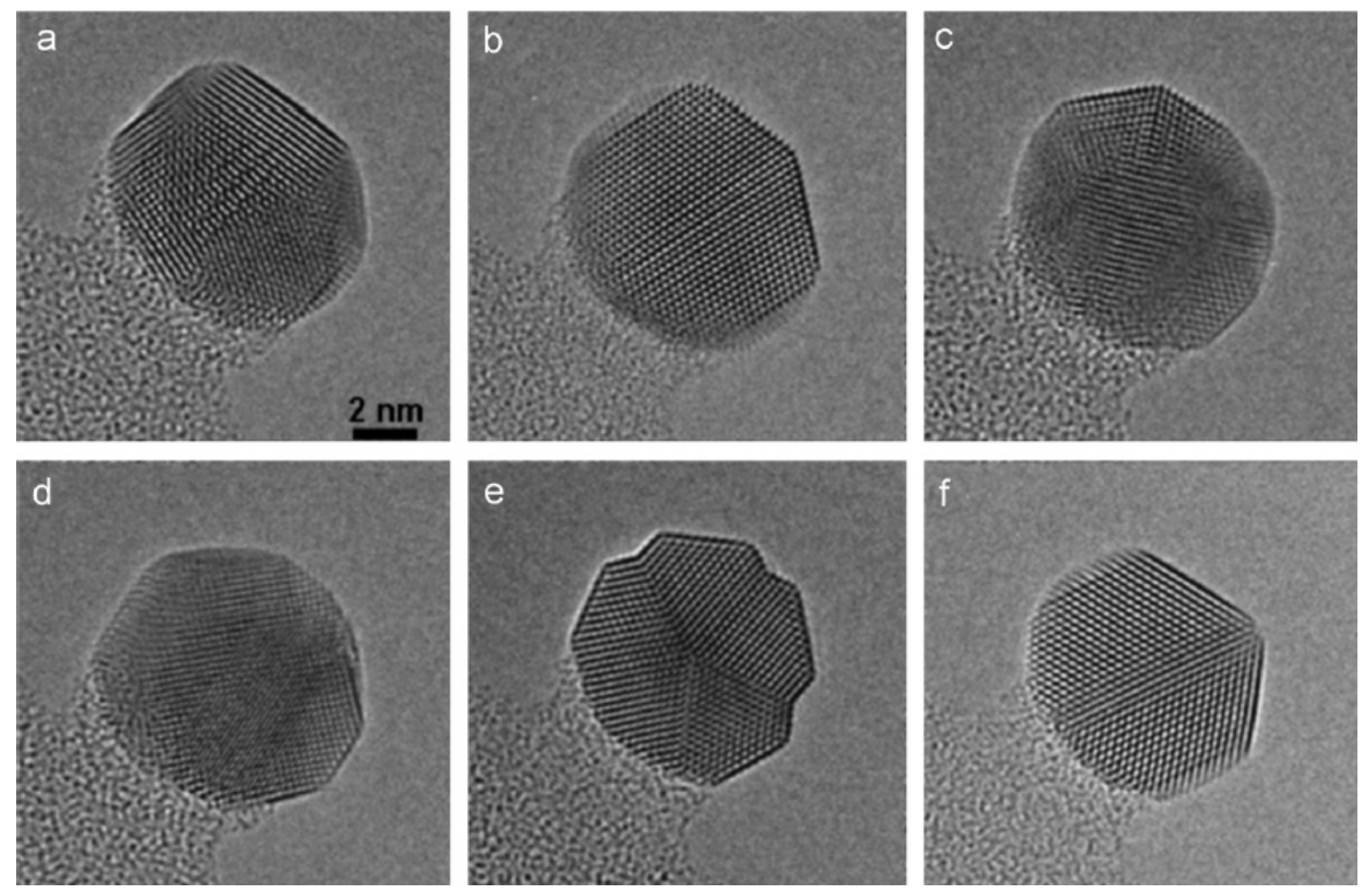

FIG.1. Controlled heating of a 5.5nm gold particle. (a) The original single crystalline fcc structure near to a $<110>$ orientation observed at room temperature. (b) At 300C, the particle structure transforms to an icosahedral structure oriented near to the $<112>$ projection. (c) Finally a decahedral structure close to $<110>$ is observed at $400 \mathrm{C}$.

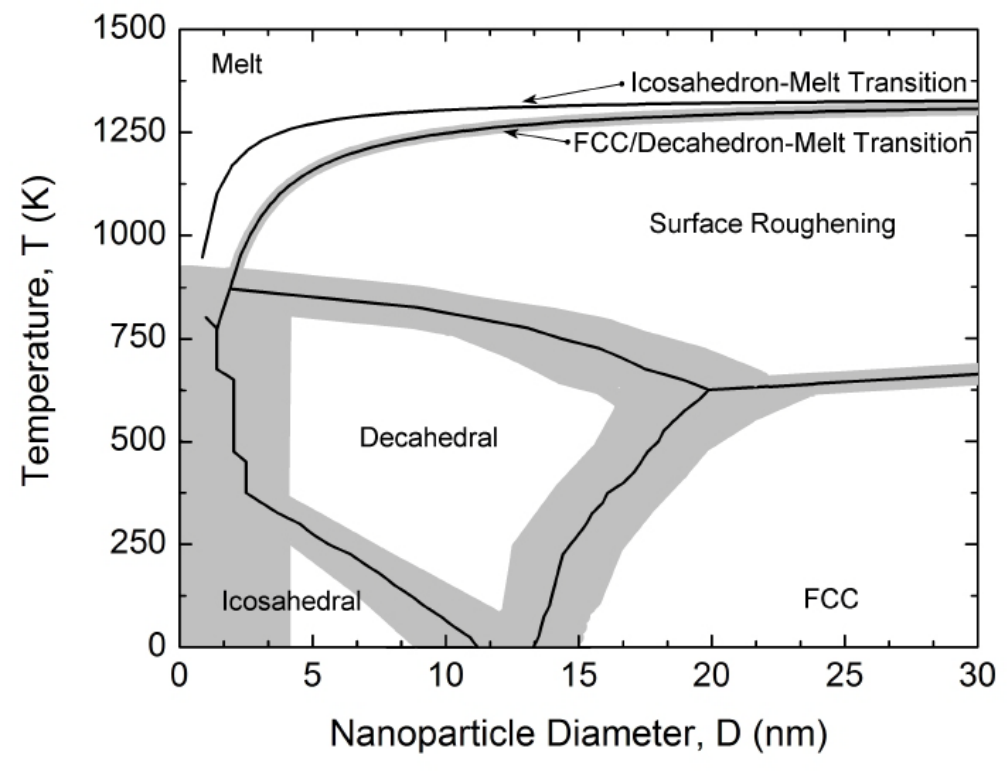

FIG.2. Quantitative phase map of gold nanoparticles, based on relativistic first principles calculations. 